Journal of Algebra Combinatorics Discrete Structures and Applications

\title{
Characterization of $2 \times 2$ nil-clean matrices over integral domains
}

Research Article

\author{
Kota Nagalakshmi Rajeswari, Umesh Gupta
}

\begin{abstract}
Let $R$ be any ring with identity. An element $a \in R$ is called nil-clean, if $a=e+n$ where $e$ is an idempotent element and $n$ is a nil-potent element. In this paper we give necessary and sufficient conditions for a $2 \times 2$ matrix over an integral domain $R$ to be nil-clean.
\end{abstract}

2010 MSC: $15 \mathrm{~A} 24$

Keywords: Nil-clean matrix, Idempotent matrix, Nil-potent matrix, Diophantine equation

\section{Introduction}

Let $R$ be an associative ring with identity. An element $a \in R$ is called clean if $a=e+u$, where $e, u \in R, e$ is an idempotent and $u$ is a unit. We shall call such a representation a clean representation of $a$. Further, $a$ is called strongly clean (uniquely clean) if it has a clean representation in which the idempotent and the unit commute (it has only one clean representation). The ring $R$ is said to be clean (strongly clean, uniquely clean) if each of its element is. The notion of clean rings was introduced by W. K. Nicholson [10] while studying rings with exchange property and observed that these rings have the exchange property. The notions of strongly clean and uniquely clean ring were introduced subsequently. Ever since, many mathematicians have studied these rings extensively.

In an attempt to unify several variants of strongly clean property A. J. Diesl [7] has introduced the notion of nil-clean rings. A ring $R$ is nil-clean if each of its elements is the sum of an idempotent and a nil-potent. The notion of strongly nil-clean (uniquely nil-clean) were defined analogous to those of strongly clean (uniquely clean). These rings have been studied in great detail in [7]. Among other things, the author has shown [[7], Prop 3.4] that a nil-clean ring is clean and immediately posed the question: "Whether or not a nil-clean element in a ring is clean?"

K. N. Rajeswari (Corresponding Author); School of Mathematics, Vigyan Bhawan, Khandwa Road, Devi Ahilya University, Indore-452001, India (email: knr_k@yahoo.co.in).

Umesh Gupta; Sanghvi Institute of Management and Science, Behind IIM, Pigdamber, Indore-453 331, India (email: ugupta19@gmail.com). 
This question was answered in negative by Andrica Dorin and Calugareanu Grigore in [3]. They have given an example of a matrix in $M_{2}(\mathbb{Z})$ which is nil-clean but not clean. In [2] they have also given example of a unit regular matrix (matrix which is the product of an idempotent matrix and a unit) which is not nil-clean and that of a nil-clean matrix which is not unit regular.

To tackle questions as above, characterization of elements having a prescribed decomposition, nilclean decomposition in the present context, are essential. As matrices are good sources of examples, it is nice to have characterization of matrices having nil-clean decomposition. Another motivation to study nil-clean matrices stems from Lie Theory, as strongly nil-clean decomposition of matrices over fields is the classical Jordan-Chevalley decomposition which is of central importance in Lie Theory.

The nil-clean (strongly nil-clean) ring theory has application in various branches of mathematics. For instance in [6] nil-cleanness of endomorphism ring of a group of finite rank and in [12] nil-cleanness of group rings have been studied.

In [4], the authors have introduced the notion of nil-clean graph of a ring $R$ for which $R$ is the set of vertices and $a, b \in R$ are adjacent if $a+b$ is nil-clean.

The characterization of nil-clean elements will certainly help understand such applications. In this paper, we give a characterization of nil-clean $2 \times 2$ matrices over an integral domain. The idempotents, unipotents (element of the form $1+n$, where $n$ is nil-potent) and nil-potents in any ring $R$ are nil-clean. By nil-clean we mean, nil-clean elements other than these.

It is well known that when $R$ is an integral domain, an idempotent $2 \times 2$ matrix other than 0 and $I$ is of the form $\left[\begin{array}{cc}x & y \\ w & 1-x\end{array}\right]$ with $x(1-x)=y w$. For the sake of completeness, a proof is given [see: Lemma 2.1].

Similarly, $2 \times 2$ nil-potent matrices are of the form $\left[\begin{array}{cc}a & b \\ c & -a\end{array}\right]$ with $a^{2}+b c=0$ [see: Lemma 2.2]. Thus a nil-clean matrix $A=\left[\begin{array}{ll}a & b \\ c & d\end{array}\right]$ satisfies the conditions

(i) $\operatorname{Tr}(A)=1$.

(ii) $\operatorname{det}\left[\begin{array}{cc}a-x & b-y \\ c-w & d-1+x\end{array}\right]=0$, where $x(1-x)=y w$.

Expanding the determinant and simplifying gives

$$
(2 a-1) x+c y+b w=a^{2}+b c .
$$

Thus, if $\left[\begin{array}{ll}a & b \\ c & d\end{array}\right]$ is nil-clean as above, then it has trace 1 and the equation (1) has a solution $x, y, w$ with $x(1-x)=y w$.

We shall show these two conditions characterize a nil-clean matrix [see: Theorem 3.1]. In [9], the authors have characterized nil-clean matrices over UFD. But, our approach is different from their approach. The authors of [9] have used characterization of rank one $2 \times 2$ matrices over a UFD $R$ to arrive at the characterization while we have used solutions of certain Diophantine equation to get the characterization.

When the entries are integers, the equation (1) is a linear Diophantine equation in 3 variables which has a solution if and only if $\operatorname{gcd}(2 a-1, c, b)$ divides $\left(a^{2}+b c\right)$ and this forces $\operatorname{gcd}(2 a-1, b, c)=1$. The condition that equation (1) has a solution satisfying $x(1-x)=y w$ yields a second degree Diophantine equation in two variables. This equation has been analyzed for its solutions in section 4 . Discussion in the sections 3 and 4 has been summarized in section 5 along with illustrations.

It has been shown [[7], Cor. 5.4] that central idempotents and central nil-potents in a ring are uniquely nil-clean. In section 6, we let $R$ be a Principal Ideal Domain (PID) and show using the characterization of section 3 that if an idempotent in $M_{2}(R)$ is not central then it is not uniquely nil-clean. Thus in $M_{2}(R)$, the only uniquely nil-clean idempotents are central idempotents i.e., the zero matrix and the identity matrix. We observe that nilpotents in $M_{2}(R)$ are uniquely nil-clean. 
Throughout the paper $R$ is an integral domain, $M_{n}(R)$ is the ring of $n \times n$ matrices over $R$. For $A \in M_{n}(R), \operatorname{Tr}(A)$ denotes the trace of $A, R(A)$ denotes its rank and $|A|$ its determinant. Let $R^{n}$ be the free $R$-module of $n$ tuples of elements of $R$.

\section{Preliminaries}

Let $R$ be an integral domain. In this section we recall some results and facts.

Lemma 2.1. $E=\left[\begin{array}{ll}x & y \\ w & z\end{array}\right] \in M_{2}(R)(E \neq I, E \neq 0)$ is idempotent if and only if it is of the form $\left[\begin{array}{cc}x & y \\ w & 1-x\end{array}\right]$ with $x(1-x)=y w$.

Proof. Let $E=\left[\begin{array}{ll}x & y \\ w & z\end{array}\right](E \neq I, E \neq 0)$ be an idempotent matrix. As idempotent matrix, which is not identity is singular, we have $x z=y w$ and as $\operatorname{Tr}(E)=R(E)=1, x+z=1$ i.e., $z=1-x$. Hence the matrix is $\left[\begin{array}{cc}x & y \\ w & 1-x\end{array}\right]$ with $x(1-x)=y w$.

Converse can be checked by direct calculation.

Lemma 2.2. $N=\left[\begin{array}{ll}a & b \\ c & d\end{array}\right] \in M_{2}(R)$ is nil-potent if and only if it is of the form $\left[\begin{array}{cc}a & b \\ c & -a\end{array}\right]$ with $a^{2}+b c=0$.

Proof. Let $N=\left[\begin{array}{ll}a & b \\ c & d\end{array}\right]$ be a nil-potent matrix. Since trace and determinant of a nil-potent matrix are always zero, $a+d=0$ and $b c-a d=0$ i.e., $d=-a$ and $b c+a^{2}=0$. Hence the matrix is $\left[\begin{array}{cc}a & b \\ c & -a\end{array}\right]$ with $b c+a^{2}=0$.

Converse is easy to check.

Proposition 2.3. The equation $x^{2}-y^{2}=c$ admits integer solutions if and only if $c$ belongs to $4 \mathbb{Z}$ or it is odd.

Proof. (See [[13], Property 1]).

Proposition 2.4. The equation $x^{2}-d y^{2}=c^{2}$ (where $d$ is not a perfect square) admits an infinite number of solutions in $\mathbb{N}$.

Proof. (See [[13], Property 2]).

Theorem 2.5. Let $R$ be a PID and $A \in M_{n}(R)$ be an idempotent matrix. Then $A$ is similar to the matrix $\left[\begin{array}{c|c}I_{r} & 0 \\ \hline 0 & 0\end{array}\right]$ where $I_{r}$ is the identity matrix of order $r$ and $r=R(A)$.

Proof. Let $B$ be a basis of $R^{n}$, the free $R$-module of rank $n$. Then $A$ determines a module homomorphism $T: R^{n} \rightarrow R^{n}$ which is idempotent and whose matrix with respect to $B$ is $A$. Let $I$ be the identity transformation, $N(T)$ and $N(I-T)$ be the null spaces of $T$ and $I-T$ respectively. As $R$ is a PID, $N(T)$ and $N(I-T)$ are free. As $T$ is idempotent, for $x \in R^{n}, T x \in N(I-T)$ and $(I-T) x \in N(T)$. Hence $R^{n}=N(I-T)+N(T)$. Clearly, $N(I-T) \cap N(T)=0$. Thus $R^{n}=N(I-T) \bigoplus N(T)$. Let $\left\{v_{1}, \ldots . v_{r}\right\}$ be a basis of $N(I-T)$ and $\left\{v_{r+1}, \ldots v_{n}\right\}$ be a basis of $N(T)$. Then $B^{\prime}=\left\{v_{1}, \ldots . v_{r}, v_{r+1}, \ldots v_{n}\right\}$ 
is a basis of $R^{n}$ and the matrix of $T$ with respect to $B^{\prime}$ is $\left[\begin{array}{c|c}I_{r} & 0 \\ \hline 0 & 0\end{array}\right]$. Therefore, $A$ is similar to $\left[\begin{array}{c|c}I_{r} & 0 \\ \hline 0 & 0\end{array}\right]$ and $r=R(A)$.

It is well known that a polynomial equation with integral coefficients for which integral solutions are desired is called a Diophantine equation. In the later part we will use two types of Diophantine equations, one linear Diophantine equation in three variables and other is quadratic Diophantine equation in two variables. A linear Diophantine equation with three variables is of the form

$$
a x+b y+c z=d
$$

where $a, b, c$ and $d$ are integers. This equation has a solution if and only if $\operatorname{gcd}(a, b, c)$ divides $d$ (see [11]). It is also that once it has a solution, it has infinite number of solutions. Solution to the equation (2) is obtained as follows (see [8]):

Let $\operatorname{gcd}(a, b)=p, a^{\prime}=a / p$ and $b^{\prime}=b / p$. Let $\left(u_{0}, v_{0}\right)$ be any one solution of $a^{\prime} u+b^{\prime} v=c,\left(z_{0}, t_{0}\right)$ be any one solution of $c z+p t=d$ and $\left(x_{0}, y_{0}\right)$ be any one solution of $a^{\prime} x+b^{\prime} y=t_{0}$, then the general solution of $a x+b y+c z=d$ is

$$
\begin{aligned}
& x=x_{0}+b^{\prime} k-u_{0} m \\
& y=y_{0}-a^{\prime} k-v_{0} m \\
& z=z_{0}+p m \quad k, m \in \mathbb{Z}
\end{aligned}
$$

We shall denote quadratic Diophantine equation in two variables by

$$
A x^{2}+B x y+C y^{2}+D x+E y+F=0
$$

where $A, B, C, D, E$ and $F$ are integers. We shall use the notations of [3] and follow the method of solution given there. Let $\mathscr{D}=B^{2}-4 A C, g=\operatorname{gcd}\left(B^{2}-4 A C, 2 A E-B D\right)$ and $\triangle=4 A C F+B D E-$ $A E^{2}-C D^{2}-F B^{2}$. Then the equation reduces to

$$
g X^{2}-\frac{\mathscr{D}}{g} Y^{2}=-4 A \frac{\triangle}{g}
$$

where $X=\frac{\mathscr{D}}{g} y+\frac{2 A E-B D}{g}$ and $Y=2 A x+B y+D$.

Now, we have the following cases:

If $\mathscr{D}>0$ and perfect square, the equation (4) admits finite number of integer solutions provided right hand side of (4) is non zero [see: Proposition 2.3].

If $\mathscr{D}>0$ and not a perfect square, the equation (4) admits an infinite number of solutions in $\mathbb{I N}[$ see: Proposition 2.4]

If $\mathscr{D}<0$ i.e., $B^{2}-4 A C<0$. (see [1]) Since the ellipse is a closed figure, the number of solutions will be finite(if a solution exists). The equation is

$$
\begin{gathered}
A x^{2}+B x y+C y^{2}+D x+E y+F=0 \\
\Rightarrow C y^{2}+(B x+E) y+\left(A x^{2}+D x+F\right)=0 \\
\Rightarrow y=\frac{-(B x+E) \pm \sqrt{(B x+E)^{2}-4 C\left(A x^{2}+D x+F\right)}}{2 C}
\end{gathered}
$$


for a value of $x$ there will be two values of $y$ except at the left and right extremes of the ellipse. In this case there will be only one value of $y$. To determine the location of the left and right extremes we should equal the square root to zero. i.e.,

$$
\begin{aligned}
& (B x+E)^{2}-4 C\left(A x^{2}+D x+F\right)=0 \\
& \Rightarrow\left(B^{2}-4 A C\right) x^{2}+2(B E-2 C D) x+\left(E^{2}-4 C F\right)=0
\end{aligned}
$$

so the value of $x$ should be between the roots of this equation. If the roots are not real, there will be no solution to the equation (5), all integer values of $x$ should be replaced in equation (6) in order to find an integer value of $y$.

\section{Main result}

Let $R$ be an integral domain. It is clear that idempotent matrices, nil-potent matrices and unit matrices of the form $I+N$, where $I$ is the identity matrix and $N$ is a nil-potent matrix are nil-clean. We shall call these trivially nil-clean. So from now onwards, nil-clean means non-trivially nil-clean. In this section, we shall discuss necessary and sufficient conditions for a matrix $A \in M_{2}(R)$ to be nil-clean.

Theorem 3.1. Let $A=\left[\begin{array}{ll}a & b \\ c & d\end{array}\right]$ be any matrix in $M_{2}(R)$. Then $A$ is nil-clean if and only if $\operatorname{Tr}(A)=1$ and $(2 a-1) x+c y+b w=a^{2}+b c$ has a solution satisfying $x(1-x)=y w$.

Proof. Let $A$ be nil-clean. Say $A=E+N$, where $E \neq 0, E \neq I d$, is an idempotent matrix and $N$ is a nil-potent matrix. Then $A-E=\left[\begin{array}{cc}a-x & b-y \\ c-w & d-1+x\end{array}\right]$ is nil-potent. Hence

$$
(A-E)^{2}=\left[\begin{array}{cc}
a-x & b-y \\
c-w & d-1+x
\end{array}\right]^{2}=0
$$

Since, the trace of a nil-potent matrix is zero, $a-x+d-1+x=0 \Rightarrow a+d=1$ i.e. $\operatorname{Tr}(A)=1$. Also, determinant of a nil-potent matrix is zero,

$$
\begin{aligned}
\text { Hence } & (a-x)(d-1+x)-(b-y)(c-w)=0 \\
\Rightarrow & (a-x)(x-a)-(b-y)(c-w)=0 \\
\Rightarrow & (a-x)^{2}+(b-y)(c-w)=0 \\
\Rightarrow & x^{2}-2 a x+a^{2}+b c-y c-b w+y w=0 \\
\Rightarrow & x^{2}-2 a x+a^{2}+b c-y c-b w+x-x^{2}=0 \\
\Rightarrow & x(1-2 a)-y c-w b+a^{2}+b c=0 \\
\Rightarrow & (2 a-1) x+c y+b w=a^{2}+b c .
\end{aligned}
$$

Conversely, let $\operatorname{Tr}(A)=1$ and $(2 a-1) x+c y+b w=a^{2}+b c$ have a solution $x, y, w$ with $x(1-x)=y w$. Let $E=\left[\begin{array}{cc}x & y \\ w & 1-x\end{array}\right]$. Then $A-E=\left[\begin{array}{cc}a-x & b-y \\ c-w & d-1+x\end{array}\right]$. Now,

$$
\begin{aligned}
\operatorname{Tr}(A-E) & =(a-x)+(d-1+x) \\
& =a+d-1 \\
& =0 \quad(\because \operatorname{Tr}(A)=1)
\end{aligned}
$$


And

$$
\begin{aligned}
|A-E| & =\left|\begin{array}{cc}
a-x & b-y \\
c-w & d-1+x
\end{array}\right| \\
& =(a-x)(d-1+x)-(b-y)(c-w) \\
& =(a-x)(x-a)-(b-y)(c-w) \\
& =-x^{2}-a^{2}+2 a x-b c+y c+b w-y w \\
& =-x^{2}-a^{2}+2 a x-b c+y c+b w-\left(x-x^{2}\right) \quad(\because y w=x(1-x)) \\
& =(2 a-1) x+c y+b w-\left(a^{2}+b c\right) \\
& =0 \quad\left[\because(2 a-1) x+c y+b w=a^{2}+b c\right]
\end{aligned}
$$

Hence $A-E$ is nil-potent, which proves $A$ is nil-clean.

\section{Analysis on main result when $R=\mathbb{Z}$}

In this section, we shall analyze the linear Diophantine equation $(2 a-1) x+c y+b w=a^{2}+b c$ as to when it has a solution satisfying $x(1-x)=y w$. Let $a_{1}=2 a-1$ and $d_{1}=a^{2}+b c$, then the equation becomes $a_{1} x+c y+b w=d_{1}$. Let $\operatorname{gcd}\left(a_{1}, c\right)=p, a_{1}^{\prime}=a_{1} / p$ and $c^{\prime}=c / p$. Let $\left(u_{0}, v_{0}\right)$ be any one solution of $a_{1}^{\prime} u+c^{\prime} v=b,\left(w_{0}, t_{0}\right)$ be any one solution of $b w+p t=d_{1}$ and $\left(x_{0}, y_{0}\right)$ be any one solution of $a_{1}^{\prime} x+c^{\prime} y=t_{0}$, then the general solution of $a_{1} x+c y+b w=d_{1}$ is

$$
\begin{aligned}
x & =x_{0}+c^{\prime} k-u_{0} m \\
y & =y_{0}-a_{1}^{\prime} k-v_{0} m \\
w & =w_{0}+p m
\end{aligned}
$$

The condition

$$
\begin{aligned}
& x-x^{2}=y w \\
\Rightarrow & \left(x_{0}+c^{\prime} k-u_{0} m\right)-\left(x_{0}+c^{\prime} k-u_{0} m\right)^{2}=\left(y_{0}-a_{1}^{\prime} k-v_{0} m\right)\left(w_{0}+p m\right) \\
\Rightarrow & \left(x_{0}+c^{\prime} k-u_{0} m\right)-\left(x_{0}^{2}+c^{\prime 2} k^{2}+u_{0}^{2} m^{2}+2 x_{0} c^{\prime} k-2 x_{0} u_{0} m-\right. \\
& \left.2 c^{\prime} k u_{0} m\right)=y_{0} w_{0}-a_{1}^{\prime} k w_{0}-v_{0} m w_{0}+y_{0} p m-a_{1}^{\prime} k p m-v_{0} m^{2} p \\
\Rightarrow & c^{\prime 2} k^{2}-\left(2 c^{\prime} u_{0}+a_{1}^{\prime} p\right) k m+\left(u_{0}^{2}-v_{0} p\right) m^{2}+\left(2 x_{0} c^{\prime}-c^{\prime}-a_{1}^{\prime} w_{0}\right) k \\
& \quad+\left(u_{0}-2 x_{0} u_{0}-v_{0} w_{0}+y_{0} p\right) m+\left(x_{0}^{2}-x_{0}+y_{0} w_{0}\right)=0 \\
\Rightarrow & A k^{2}+B k m+C m^{2}+D k+E m+F=0 \\
& \text { where } A=c^{\prime 2}, B=-\left(2 c^{\prime} u_{0}+a_{1}^{\prime} p\right), C=\left(u_{0}^{2}-v_{0} p\right), D=\left(2 x_{0} c^{\prime}-c^{\prime}\right. \\
& \left.-a_{1}^{\prime} w_{0}\right), E=\left(u_{0}-2 x_{0} u_{0}-v_{0} w_{0}+y_{0} p\right), \text { and } F=\left(x_{0}^{2}-x_{0}+y_{0} w_{0}\right)
\end{aligned}
$$

Now,

$$
\begin{aligned}
\mathscr{D} & =B^{2}-4 A C \\
= & \left(2 c^{\prime} u_{0}+a_{1}^{\prime} p\right)^{2}-4 c^{\prime 2}\left(u_{0}^{2}-v_{0} p\right) \\
= & 4 c^{\prime 2} u_{0}^{2}+a_{1}^{\prime 2} p^{2}+4 c^{\prime} u_{0} a_{1}^{\prime} p-4 c^{\prime 2} u_{0}^{2}+4 c^{\prime 2} v_{0} p \\
= & a_{1}^{2}+4 c^{\prime} u_{0} a_{1}^{\prime} p+4 c^{\prime 2} v_{0} p \\
= & a_{1}^{2}+4 c^{\prime} u_{0} a_{1}^{\prime} p+4 c^{\prime} p\left(b-a_{1}^{\prime} u_{0}\right) \quad\left(\because a_{1}^{\prime} u_{0}+c^{\prime} v_{0}=b\right) \\
= & a_{1}^{2}+4 c b . \\
2 A E-B D= & 2 c^{\prime 2}\left(u_{0}-2 x_{0} u_{0}-v_{0} w_{0}+y_{0} p\right)+\left(2 c^{\prime} u_{0}+a_{1}^{\prime} p\right)\left(2 x_{0} c^{\prime}-c^{\prime}\right. \\
& \left.-a_{1}^{\prime} w_{0}\right)
\end{aligned}
$$




$$
\begin{aligned}
&= 2 c^{\prime 2} u_{0}-4 c^{\prime 2} x_{0} u_{0}-2 c^{\prime 2} v_{0} w_{0}+2 c^{\prime 2} y_{0} p+4 c^{\prime 2} u_{0} x_{0}-2 c^{2} u_{0} \\
&-2 c^{\prime} u_{0} a_{1}^{\prime} w_{0}+2 a_{1}^{\prime} p x_{0} c^{\prime}-a_{1}^{\prime} p c^{\prime}-a_{1}^{\prime 2} p w_{0} \\
&=-2 c^{\prime} w_{0}\left(a_{1}^{\prime} u_{0}+c^{\prime} v_{0}\right)+2 c^{\prime} p\left(a_{1}^{\prime} x_{0}+c^{\prime} y_{0}\right)-a_{1}^{\prime} p c^{\prime}-a_{1}^{\prime 2} p w_{0} \\
&=-2 c^{\prime} w_{0} b+2 c^{\prime} p t_{0}-a_{1}^{\prime} p c^{\prime}-a_{1}^{\prime 2} p w_{0}\left(\begin{array}{c}
a_{1}^{\prime} x_{0}+c^{\prime} y_{0}=t_{0} \\
a_{1}^{\prime} u_{0}+c^{\prime} v_{0}=b
\end{array}\right) \\
&=-2 c^{\prime} w_{0} b+2 c^{\prime}\left(d_{1}-b w_{0}\right)-a_{1}^{\prime} p c^{\prime}-a_{1}^{\prime 2} p w_{0} \\
&=2 c^{\prime} d_{1}-4 c^{\prime} w_{0} b-a_{1}^{\prime} p c^{\prime}-a_{1}^{\prime 2} p w_{0} \\
&=\frac{1}{p}\left(2 c d_{1}-4 c w_{0} b-a_{1} c-a_{1}^{2} w_{0}\right)
\end{aligned}
$$

Let $g=\operatorname{gcd}(\mathscr{D}, 2 A E-B D)$.

$$
\begin{aligned}
& \triangle=4 A C F+B D E-A E^{2}-C D^{2}-F B^{2} \\
& =4 c^{\prime 2}\left(u_{0}^{2}-v_{0} p\right)\left(x_{0}^{2}-x_{0}+y_{0} w_{0}\right)-\left(2 c^{\prime} u_{0}+a_{1}^{\prime} p\right)\left(2 x_{0} c^{\prime}-c^{\prime}-a_{1}^{\prime} w_{0}\right) \\
& \left(u_{0}-2 x_{0} u_{0}-v_{0} w_{0}+y_{0} p\right)-c^{2}\left(u_{0}-2 x_{0} u_{0}-v_{0} w_{0}+y_{0} p\right)^{2}-\left(u_{0}^{2}\right. \\
& \left.-v_{0} p\right)\left(2 x_{0} c^{\prime}-c^{\prime}-a_{1}^{\prime} w_{0}\right)^{2}-\left(x_{0}^{2}-x_{0}+y_{0} w_{0}\right)\left(2 c^{\prime} u_{0}+a_{1}^{\prime} p\right)^{2} \\
& =4 c^{\prime 2} u_{0}^{2} x_{0}^{2}-4 c^{\prime 2} u_{0}^{2} x_{0}+4 c^{\prime 2} u_{0}^{2} y_{0} w_{0}-4 c^{\prime 2} v_{0} p x_{0}^{2}+4 c^{\prime 2} v_{0} p x_{0}-4 c^{\prime 2} v_{0} p y_{0} w_{0} \\
& -4 c^{\prime 2} x_{0} u_{0}^{2}+2 c^{\prime 2} u_{0}^{2}+2 c^{\prime} a_{1}^{\prime} w_{0} u_{0}^{2}+8 c^{\prime 2} u_{0}^{2} x_{0}^{2}-4 c^{\prime 2} u_{0}^{2} x_{0}-4 c^{\prime} u_{0}^{2} a_{1}^{\prime} w_{0} x_{0} \\
& +4 c^{\prime 2} u_{0} v_{0} w_{0} x_{0}-2 c^{\prime 2} u_{0} v_{0} w_{0}-2 c^{\prime} u_{0} a_{1}^{\prime} w_{0}^{2} v_{0}-4 c^{\prime 2} u_{0} y_{0} p x_{0}+2 c^{\prime 2} u_{0} y_{0} p \\
& +2 c^{\prime} u_{0} a_{1}^{\prime} y_{0} p w_{0}-2 a_{1}^{\prime} p x_{0} c^{\prime} u_{0}+a_{1}^{\prime} p c^{\prime} u_{0}+a_{1}^{\prime 2} p w_{0} u_{0}+4 a_{1}^{\prime} x_{0}^{2} c^{\prime} u_{0} p \\
& -2 a_{1}^{\prime} p c^{\prime} x_{0} u_{0}-2 a_{1}^{\prime 2} p w_{0} x_{0} u_{0}+2 a_{1}^{\prime} p v_{0} w_{0} x_{0} c^{\prime}-a_{1}^{\prime} p c^{\prime} v_{0} w_{0}-a_{1}^{\prime 2} w_{0}^{2} v_{0} p \\
& -2 a_{1}^{\prime} p^{2} y_{0} x_{0} c^{\prime}+a_{1}^{\prime} p^{2} c^{\prime} y_{0}+a_{1}^{\prime 2} p^{2} y_{0} w_{0}-c^{\prime 2} u_{0}^{2}-4 c^{\prime 2} x_{0}^{2} u_{0}^{2}-c^{\prime 2} v_{0}^{2} w_{0}^{2} \\
& -c^{\prime 2} y_{0}^{2} p^{2}+4 c^{\prime 2} x_{0} u_{0}^{2}+2 c^{\prime 2} u_{0} v_{0} w_{0}-2 c^{\prime 2} u_{0} y_{0} p-4 c^{\prime 2} x_{0} u_{0} v_{0} w_{0} \\
& +4 c^{\prime 2} x_{0} u_{0} y_{0} p+2 c^{\prime 2} v_{0} w_{0} y_{0} p-4 u_{0}^{2} x_{0}^{2} c^{\prime 2}-u_{0}^{2} c^{\prime 2}-u_{0}^{2} a_{1}^{\prime 2} w_{0}^{2}+4 u_{0}^{2} x_{0} c^{\prime 2} \\
& +4 u_{0}^{2} x_{0} c^{\prime} a_{1}^{\prime} w_{0}-2 u_{0}^{2} c^{\prime} a_{1}^{\prime} w_{0}+4 v_{0} p x_{0}^{2} c^{\prime 2}+c^{\prime 2} v_{0} p+a_{1}^{\prime 2} w_{0}^{2} p v_{0}-4 c^{\prime 2} x_{0} v_{0} p \\
& -4 c^{\prime} a_{1}^{\prime} w_{0} x_{0} v_{0} p+2 c^{\prime} a_{1}^{\prime} w_{0} v_{0} p-4 x_{0}^{2} c^{\prime 2} u_{0}^{2}-x_{0}^{2} a_{1}^{\prime 2} p^{2}-4 c^{\prime} a_{1}^{\prime} x_{0}^{2} u_{0} p+4 c^{2} u_{0}^{2} x_{0} \\
& +a_{1}^{\prime 2} p^{2} x_{0}+4 c^{\prime} a_{1}^{\prime} u_{0} x_{0} p-4 c^{\prime 2} u_{0}^{2} y_{0} w_{0}-a_{1}^{\prime 2} p^{2} y_{0} w_{0}-4 c^{\prime} a_{1}^{\prime} u_{0} y_{0} w_{0} p \\
& =\left(4 c^{\prime 2} u_{0}^{2} x_{0}^{2}+8 c^{\prime 2} u_{0}^{2} x_{0}^{2}-4 c^{\prime 2} u_{0}^{2} x_{0}^{2}-4 c^{\prime 2} u_{0}^{2} x_{0}^{2}-4 c^{\prime 2} u_{0}^{2} x_{0}^{2}\right)+\left(-4 c^{\prime 2} u_{0}^{2} x_{0}\right. \\
& \left.-4 c^{\prime 2} u_{0}^{2} x_{0}-4 c^{2} u_{0}^{2} x_{0}+4 c^{2} u_{0}^{2} x_{0}+4 c^{\prime 2} u_{0}^{2} x_{0}+4 c^{\prime 2} u_{0}^{2} x_{0}\right)+\left(4 c^{2} u_{0}^{2} y_{0} w_{0}\right. \\
& \left.-4 c^{\prime 2} u_{0}^{2} y_{0} w_{0}\right)+\left(4 c^{\prime 2} v_{0} p x_{0}^{2}-4 c^{\prime 2} v_{0} p x_{0}^{2}\right)+\left(4 c^{\prime 2} v_{0} p x_{0}-4 c^{\prime 2} v_{0} p x_{0}\right) \\
& +\left(2 c^{\prime 2} v_{0} p y_{0} w_{0}-4 c^{\prime 2} v_{0} p y_{0} w_{0}\right)+\left(2 c^{\prime 2} u_{0}^{2}-c^{\prime 2} u_{0}^{2}-c^{\prime 2} u_{0}^{2}\right)+\left(2 c^{\prime} a_{1}^{\prime} w_{0} u_{0}^{2}\right. \\
& \left.-2 c^{\prime} a_{1}^{\prime} w_{0} u_{0}^{2}\right)+\left(4 c^{\prime} u_{0}^{2} a_{1}^{\prime} w_{0} x_{0}-4 c^{\prime} u_{0}^{2} a_{1}^{\prime} w_{0} x_{0}\right)+\left(4 c^{\prime 2} u_{0} v_{0} w_{0} x_{0}\right. \\
& \left.-4 c^{\prime 2} u_{0} v_{0} w_{0} x_{0}\right)+\left(2 c^{\prime 2} u_{0} v_{0} w_{0}-2 c^{\prime 2} u_{0} v_{0} w_{0}\right)-2 c^{\prime} u_{0} a_{1}^{\prime} w_{0}^{2} v_{0}+\left(4 c^{\prime 2} u_{0} y_{0} p x_{0}\right. \\
& \left.-4 c^{\prime 2} u_{0} y_{0} p x_{0}\right)+\left(2 c^{\prime 2} u_{0} y_{0} p-2 c^{\prime 2} u_{0} y_{0} p\right)+\left(2 c^{\prime} u_{0} a_{1}^{\prime} y_{0} p w_{0}-4 c^{\prime} u_{0} a_{1}^{\prime} y_{0} p w_{0}\right) \\
& +\left(4 a_{1}^{\prime} p x_{0} c^{\prime} u_{0}-2 a_{1}^{\prime} p x_{0} c^{\prime} u_{0}-2 a_{1}^{\prime} p x_{0} c^{\prime} u_{0}\right)+a_{1}^{\prime} p c^{\prime} u_{0}+a_{1}^{\prime 2} p w_{0} u_{0} \\
& +\left(4 a_{1}^{\prime} x_{0}^{2} c^{\prime} u_{0} p-4 a_{1}^{\prime} x_{0}^{2} c^{\prime} u_{0} p\right)-2 a_{1}^{\prime 2} p w_{0} x_{0} u_{0}+\left(2 a_{1}^{\prime} p v_{0} w_{0} x_{0} c^{\prime}-\right. \\
& \left.4 a_{1}^{\prime} p v_{0} w_{0} x_{0} c^{\prime}\right)-a_{1}^{\prime} p c^{\prime} v_{0} w_{0}+\left(a_{1}^{\prime 2} w_{0}^{2} v_{0} p-a_{1}^{\prime 2} w_{0}^{2} v_{0} p\right)-2 a_{1}^{\prime} p^{2} y_{0} x_{0} c^{\prime}
\end{aligned}
$$




$$
\begin{aligned}
& +a_{1}^{\prime} p^{2} c^{\prime} y_{0}+\left(a_{1}^{\prime 2} p^{2} y_{0} w_{0}-a_{1}^{\prime 2} p^{2} y_{0} w_{0}\right)-c^{\prime 2} v_{0}^{2} w_{0}^{2}-c^{\prime 2} y_{0}^{2} p^{2}-u_{0}^{2} a_{1}^{\prime 2} w_{0}^{2} \\
& +c^{\prime 2} v_{0} p+2 c^{\prime} a_{1}^{\prime} w_{0} v_{0} p-x_{0}^{2} p^{2} a_{1}^{\prime 2}+a_{1}^{\prime 2} p^{2} x_{0} \\
= & -2 c^{\prime 2} v_{0} p y_{0} w_{0}-2 c^{\prime} u_{0} a_{1}^{\prime} w_{0}^{2} v_{0}-2 c^{\prime} a_{1}^{\prime} u_{0} y_{0} w_{0} p+a_{1}^{\prime} p c^{\prime} u_{0}+a_{1}^{\prime 2} p w_{0} u_{0} \\
& -2 a_{1}^{\prime 2} p w_{0} x_{0} u_{0}-2 c^{\prime} a_{1}^{\prime} p w_{0} v_{0} x_{0}-a_{1}^{\prime} p c^{\prime} v_{0} w_{0}-2 a_{1}^{\prime} p^{2} y_{0} x_{0} c^{\prime}+a_{1}^{\prime} p^{2} c^{\prime} y_{0} \\
& -c^{\prime 2} v_{0}^{2} w_{0}^{2}-c^{\prime 2} y_{0}^{2} p^{2}-u_{0}^{2} a_{1}^{\prime 2} w_{0}^{2}+c^{\prime 2} v_{0} p+2 c^{\prime} a_{1}^{\prime} w_{0} v_{0} p-x_{0}^{2} p^{2} a_{1}^{\prime 2}+a_{1}^{\prime 2} p^{2} x_{0} \\
= & -\left(c^{\prime 2} y_{0}^{2} p^{2}+c^{\prime 2} v_{0}^{2} w_{0}^{2}+2 c^{\prime 2} v_{0} p y_{0} w_{0}\right)-\left(x_{0}^{2} p^{2} a_{1}^{\prime 2}+u_{0}^{2} a_{1}^{\prime 2} w_{0}^{2}+2 a_{1}^{\prime 2} p x_{0} u_{0} w_{0}\right) \\
& -2\left(a_{1}^{\prime} p^{2} y_{0} x_{0} c^{\prime}+c^{\prime} a_{1}^{\prime} y_{0} u_{0} w_{0} p+c^{\prime} v_{0} w_{0} x_{0} p a_{1}^{\prime}+c^{\prime} u_{0} w_{0}^{2} v_{0} a_{1}^{\prime}\right)+a_{1}^{\prime} p c^{\prime} u_{0} \\
& +a_{1}^{\prime 2} p w_{0} u_{0}+a_{1}^{\prime} p^{2} c^{\prime} y_{0}+c^{\prime 2} v_{0} p+c^{\prime} a_{1}^{\prime} w_{0} v_{0} p+a_{1}^{\prime 2} p^{2} x_{0} . \\
= & -\left(c^{\prime} y_{0} p+c^{\prime} v_{0} w_{0}+x_{0} p a_{1}^{\prime}+u_{0} a_{1}^{\prime} w_{0}\right)^{2}+c^{\prime} p\left(a_{1}^{\prime} u_{0}+c^{\prime} v_{0}\right)+a_{1}^{\prime} p w_{0} \\
& \left(a_{1}^{\prime} u_{0}+c^{\prime} v_{0}\right)+a_{1}^{\prime} p^{2}\left(a_{1}^{\prime} x_{0}+c^{\prime} y_{0}\right) \\
= & -\left[p\left(a_{1}^{\prime} x_{0}+c^{\prime} y_{0}\right)+w_{0}\left(c^{\prime} v_{0}+a_{1}^{\prime} u_{0}\right)\right]^{2}+c^{\prime} p b+a_{1}^{\prime} p w_{0} b \\
& +a_{1}^{\prime} p^{2} t_{0} \quad\left[\because a_{1}^{\prime} u_{0}+c^{\prime} v_{0}=b\right. \\
= & -\left(p t_{0}+b w_{0}\right)^{2}+c b+a_{1}^{\prime} p\left(b w_{0}+p t_{0}\right) \\
= & -d_{1}^{2}+c b+a_{1} d_{1} \\
= & -\left(a^{2}+b c\right)^{2}+(2 a-1)\left(a^{2}+b c\right)+b c \\
= & -\left(a^{2}+b c\right)^{2}+2 a\left(a^{2}+b c\right)-a^{2}-b c+b c \\
= & -\left(a^{2}+b c-a\right)^{2} \\
= & \left.-\left(d_{1}-a\right)^{2} \quad d_{1}\right)
\end{aligned}
$$

The equation (7) reduces to $g X^{2}-\frac{\mathscr{Q}}{g} Y^{2}=-4 A \frac{\Delta}{g}$

$$
\text { Or } \quad X_{1}^{2}-\mathscr{D} Y^{2}=\left[2 c^{\prime}\left(d_{1}-a\right)\right]^{2}
$$

Where $X_{1}=g X=\mathscr{D} m+(2 A E-B D)=\left(a_{1}^{2}+4 b c\right) m+\frac{1}{p}\left(2 c d_{1}-4 c w_{0} b-a_{1} c-a_{1}^{2} w_{0}\right)$ and $Y=$ $2 A k+B m+D=2 c^{\prime 2} k-\left(2 c^{\prime} u_{0}+a_{1}\right) m+\left(2 x_{0} c^{\prime}-c^{\prime}-a_{1}^{\prime} w_{0}\right)$. The general methods of solutions of (8) have already been discussed in section 2 .

\section{Examples}

In this section, we shall briefly summarize the discussion in section 3 and 4 . For a given matrix $A=\left[\begin{array}{ll}a & b \\ c & d\end{array}\right]$ to be nil-clean the following conditions are necessary and sufficient :

(1) $\operatorname{Tr}(A)=1$

(2) $\operatorname{gcd}(2 a-1, b, c)=1$

(3) The equation (8) of section 4 has a solution.

The existence of solutions and the number of solutions is determined by the discriminant $\mathscr{D}$ of the equation (8) which is $(2 a-1)^{2}+b c$.

(i) If $\mathscr{D}>0$, a perfect square and right hand side of (8) is non zero, then it has finitely many solutions (see Example 5.1 below).

(ii) If $\mathscr{D}>0$ and not a perfect square (8) has infinitely many solutions (see Example 5.2 below).

(iii) If $\mathscr{D}<0$, equation (8) may or may not have a solution. If (8) has a solution then it has finitely many (see Example 5.3 below). Finally, Example 5.4 illustrates the case where (8) does not have a solution. 
Example 5.1. Consider the matrix $A=\left[\begin{array}{ll}1 & 3 \\ 2 & 0\end{array}\right]$. The linear Diophantine equation $(2 a-1) x+c y+b w=$ $a^{2}+b c$ for $A$ is $x+2 y+3 w=7$. The solutions are $x=1+2 k-m, y=3-k-m, w=m$ where $k$ and $m$ are integers. Using condition $x(1-x)=y w$, we get the quadratic Diophantine equation $4 k^{2}-5 k m+2 k+2 m=0$. Here $\mathscr{D}=25$ which is square of 5 and solutions are $(4,4),(-2,-1),(0,0)$ and $(1,2)$. For the solution $(4,4)$, we get the solution of linear Diophantine equation $x=5, y=-5, w=4$. Let $E=\left[\begin{array}{ll}5 & -5 \\ 4 & -4\end{array}\right]$. Then $A-E$ is nil-potent.

Example 5.2. Consider the matrix $A=\left[\begin{array}{cc}2 & 3 \\ 2 & -1\end{array}\right]$. The linear Diophantine equation $(2 a-1) x+c y+$ $b w=a^{2}+b c$ for $A$ is $3 x+2 y+3 w=10$. The solutions are $x=2 k-m, y=5-3 k, w=m$ where $k$ and $m$ are integers. Using condition $x(1-x)=y w$, we get the quadratic Diophantine equation $4 k^{2}-7 k m+m^{2}-2 k+6 m=0$. Here $\mathscr{D}=33$ which is not a perfect square and this equation has infinite number of solutions, one of the solution is (2, 2) for which, we get the solution of linear Diophantine equation $x=2, y=-1, w=2$. Let $E=\left[\begin{array}{ll}2 & -1 \\ 2 & -1\end{array}\right]$. Then $A-E$ is nil-potent.

Example 5.3. Consider the matrix $A=\left[\begin{array}{cc}2 & 3 \\ -1 & -1\end{array}\right]$ The linear Diophantine equation $(2 a-1) x+c y+b w=$ $a^{2}+b c$ for $A$ is $3 x-y+3 w=1$. The solutions are $x=-k, y=-1-3 k+3 m, w=m$ where $k$ and $m$ are integers. Using condition $x(1-x)=y w$, we get the quadratic Diophantine equation $k^{2}-3 k m+3 m^{2}+k-m=0$. Here $\mathscr{D}=-3<0$ and solutions are $(-2,-1),(-1,0)$ and $(0,0)$. For the solution (-2, -1), we get the solution of linear Diophantine equation $x=2, y=2, w=-1$. Let $E=\left[\begin{array}{cc}2 & 2 \\ -1 & -1\end{array}\right]$. Then $A-E$ is nil-potent.

Example 5.4. Consider the matrix $A=\left[\begin{array}{cc}2 & 3 \\ -5 & -1\end{array}\right]$. The linear Diophantine equation $(2 a-1) x+c y+$ $b w=a^{2}+b c$ for $A$ is $3 x-5 y+3 w=-11$. The solutions are $x=3-5 k-m, y=4-3 k, w=m$ where $k$ and $m$ are integers. Using condition $x(1-x)=y w$, we get the quadratic Diophantine equation $25 k^{2}+7 k m+m^{2}-25 k-m+6=0$. Here $\mathscr{D}=-51<0$. This equation has no solution as the equation $\left(B^{2}-4 A C\right) k^{2}+2(B E-2 C D) k+\left(E^{2}-4 C F\right)=0$ i.e., $51 k^{2}-86 k+23=0$ has roots 0.33 and 1.35 and the integer $k=1$ does not yield integer value for $m$. Hence $3 x-5 y+3 w=-11$ does not have a solution satisfying $x(1-x)=y w$.

\section{Uniquely nil-clean matrices}

In [7], it has been shown that central idempotents and central nilpotents are uniquely nil-clean. When $R$ is a PID, we show that only uniquely nil-clean idempotent elements of $M_{2}(R)$ are central idempotents and observe that all nilpotents in $M_{2}(R)$ are uniquely nil-clean.

Remark 6.1. Nil-clean property is preserved under similarity.

Proof. Let $A$ be nil-clean and $A=E+N$, where $E$, an idempotent matrix and $N$, a nilpotent matrix. Say $N^{k}=0$. Let $B$ be similar to $A$. Then $B=C A C^{-1}$ for some invertible matrix $C$. Therefore, $B=C E C^{-1}+C N C^{-1}$, where $\left(C E C^{-1}\right)^{2}=C E^{2} C^{-1}=C E C^{-1}$ and $\left(C N C^{-1}\right)^{k}=C N^{k} C^{-1}=0$. Hence $B$ is nil-clean.

Lemma 6.2. Let $R$ be a PID and $E \in M_{2}(R)$ be an idempotent which is not central. Then $E$ is not uniquely nil-clean. 
Proof. Since $E$ is not central, $E \neq 0$ and $E \neq I$. Then $E=\left[\begin{array}{cc}x & y \\ w & 1-x\end{array}\right]$, where $y w=x(1-x)$. As every idempotent matrix of rank $r$ over a PID is similar to the matrix of the form $\left[\begin{array}{c|c}I_{r} & 0 \\ \hline 0 & 0\end{array}\right]$ (By Theorem 2.5), $E$ is similar to $\left[\begin{array}{ll}1 & 0 \\ 0 & 0\end{array}\right]$. Let $C E C^{-1}=\left[\begin{array}{ll}1 & 0 \\ 0 & 0\end{array}\right]$. The equation $(2 a-1) x+c y+b w=a^{2}+b c$ of section 3 , for $\left[\begin{array}{ll}1 & 0 \\ 0 & 0\end{array}\right]$ reduces to $x=1$. As we are interested in the solution $x, y$, with $y w=x(1-x)$, we have $y w=0$. Hence either $y=0$ and $w$ is arbitrary or $y$ is arbitrary and $w=0$. Hence all the nil-clean decomposition of $\left[\begin{array}{ll}1 & 0 \\ 0 & 0\end{array}\right]$ are

$\left[\begin{array}{ll}1 & 0 \\ 0 & 0\end{array}\right]=\left[\begin{array}{ll}1 & 0 \\ w & 0\end{array}\right]+\left[\begin{array}{cc}0 & 0 \\ -w & 0\end{array}\right], w$ is arbitrary

and $\left[\begin{array}{ll}1 & 0 \\ 0 & 0\end{array}\right]=\left[\begin{array}{ll}1 & y \\ 0 & 0\end{array}\right]+\left[\begin{array}{cc}0 & -y \\ 0 & 0\end{array}\right], y$ is arbitrary.

Therefore, $E=C^{-1}\left[\begin{array}{ll}1 & 0 \\ w & 0\end{array}\right] C+C^{-1}\left[\begin{array}{cc}0 & 0 \\ -w & 0\end{array}\right] C, w$ is arbitrary

and $E=C^{-1}\left[\begin{array}{ll}1 & y \\ 0 & 0\end{array}\right] C+C^{-1}\left[\begin{array}{cc}0 & -y \\ 0 & 0\end{array}\right] C, y$ is arbitrary.

Hence $E$ is not uniquely nil-clean.

Example 6.3. Let $R=\mathbb{Z}$ and let $A=\left[\begin{array}{cc}9 & -6 \\ 12 & -8\end{array}\right]$ and $C=\left[\begin{array}{cc}3 & -2 \\ -4 & 3\end{array}\right]$ then $C^{-1} A C=\left[\begin{array}{ll}1 & 0 \\ 0 & 0\end{array}\right]$. The nil-clean decomposition of $\left[\begin{array}{ll}1 & 0 \\ 0 & 0\end{array}\right]$ is

$$
\begin{aligned}
{\left[\begin{array}{ll}
1 & 0 \\
0 & 0
\end{array}\right]=\left[\begin{array}{ll}
1 & 0 \\
w & 0
\end{array}\right]+} & {\left[\begin{array}{cc}
0 & 0 \\
-w & 0
\end{array}\right] \text { where } w \text { is arbitrary. Therefore, } } \\
{\left[\begin{array}{cc}
9 & -6 \\
12 & -8
\end{array}\right] } & =\left[\begin{array}{ll}
3 & 2 \\
4 & 3
\end{array}\right]\left(\left[\begin{array}{ll}
1 & 0 \\
w & 0
\end{array}\right]+\left[\begin{array}{cc}
0 & 0 \\
-w & 0
\end{array}\right]\right)\left[\begin{array}{cc}
3 & -2 \\
-4 & 3
\end{array}\right] \\
& =\left(\left[\begin{array}{ll}
3+2 w & 0 \\
4+3 w & 0
\end{array}\right]+\left[\begin{array}{cc}
-2 w & 0 \\
-3 w & 0
\end{array}\right]\right)\left[\begin{array}{cc}
3 & -2 \\
-4 & 3
\end{array}\right] \\
& =\left[\begin{array}{cc}
9+6 w & -6-4 w \\
12+9 w & -8-6 w
\end{array}\right]+\left[\begin{array}{cc}
-6 w & 4 w \\
-9 w & 6 w
\end{array}\right] \text { where } w \in \mathbb{Z} \text { is arbitrary }
\end{aligned}
$$

Hence $A$ is not uniquely nil-clean.

Lemma 6.4. If $A \in M_{2}(R)$ is nilpotent, then it is uniquely nil-clean.

Proof. Let $A \in M_{2}(R)$ be nilpotent. Suppose $A=E+N$, where $E$, an idempotent matrix and $N$, a nilpotent matrix, is a nil-clean representation of $A$. Comparing the traces on both sides, we have $\operatorname{Tr}(E)=0$. Therefore, $E=0$ and $A=N$.

In [5], the notion of nil-clean elements has been generalized to ideals of a ring $R$. An ideal $I$ of $R$ is nil-clean if $I=E+N$, where $E$ and $N$ are ideals of $R, E^{2}=E$ and $N$ is a nil ideal(i.e., all elements of $N$ 
are nil-potent). While the ideals generated by idempotents are idempotent, those generated by nilpotent elements need not be nil or nil-potent when the ring $R$ is non-commutative.

In view of the results of the paper, we ask the following:

Question 6.4. If $I$ is the principal ideal generated by a nil-clean element in $M_{2}(R), R$ an integral domain, when is I nil-clean?

Acknowledgment: The authors thank the referees immensely for their suggestions which have improved the earlier version of the paper.

\section{References}

[1] D. Alpern, Generic Two Integer Variable Equation Solver, 2018, available at www.alpertron.com.ar/QUAD.HTM.

[2] T. Andreescu, D. Andrica, Quadratic Diophantine Equations, Springer, New York, 2015.

[3] D. Andrica, G. Călugăreanu, A nil-clean $2 \times 2$ matrix over the integers which is not clean, J. Algebra Appl. 13(6) (2014) 1450009.

[4] D. K. Basnet, J. Bhattacharyya, Nil clean graph of rings, arXiv:1701.07630 [math.RA], https://arxiv.org/abs/1701.07630.

[5] A. T. Block Gorman, Generalizations of Nil Clean to Ideals, Wellesley College, Honors Thesis Collection, (388) 2016.

[6] S. Breaz, G. Călugăreanu, P. Danchev, T. Micu, Nil-clean matrix rings, Linear Algebra Appl. 439(10) (2013) 3115-3119.

[7] A. J. Diesl, Nil-clean rings, J. Algebra 383(1) (2013) 197-211.

[8] Diophantine Equation $a x+b y+c z=d$ Solver, www.mathafou.free.fr/ex e_en/exedioph3.html.

[9] S. Hadjirezaei, S. Karimzadeh, On the nil-clean matrix over a UFD, J. Alg. Struc. Appl. 2(2) (2015) 49-55.

[10] W. K. Nicholson, Lifting idempotents and exchange rings, Trans. Amer. Math. Soc. 229 (1977) 269-278.

[11] I. Niven, H. S. Zuckerman, An Introduction to the Theory of Numbers, John Wiley-Sons, 3rd edition, 1972.

[12] S. Sahinkaya, G. Tang, Y. Zhou, Nil-clean group rings, J. Algebra Appl. 16(7) (2017) 1750135.

[13] F. Smarandache, Existence and number of solutions of Diophantine quadratic equations with two unkowns in $\mathbb{Z}$ and $\mathrm{N}$, arXiv:0 704.3716 [math.GM], http://arxiv.org/abs/0704.3716. 